The Development of Students' use of Additive and Proportional Methods along Primary and Secondary School

Ceneida Fernández, Salvador Llinares, Wim Van Dooren, Dirk De Bock en Lieven Verschaffel

HUB RESEARCH PAPER 2010/32 NOVEMBER 2010

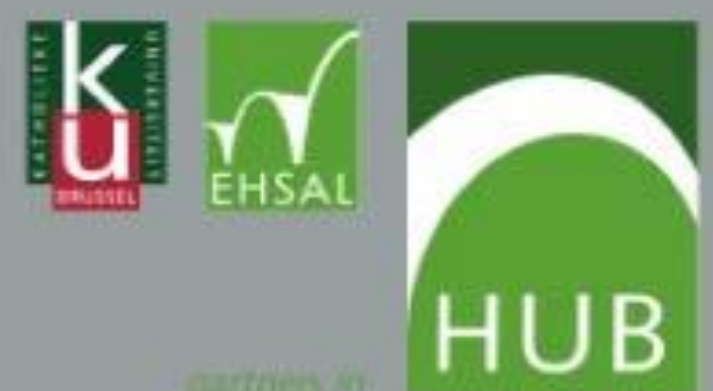




\title{
THE DEVELOPMENT OF STUDENTS' USE OF ADDITIVE AND PROPORTIONAL METHODS ALONG PRIMARY AND SECONDARY SCHOOL
}

\author{
Ceneida Fernández ${ }^{1}$; Salvador Llinares ${ }^{1}$; Wim Van Dooren ${ }^{2}$; Dirk De Bock ${ }^{3}$ and \\ Lieven Verschaffel ${ }^{2}$ \\ ${ }^{1}$ Universidad de Alicante, Spain \\ ${ }^{2}$ Katholieke Universiteit Leuven, Belgium \\ ${ }^{3}$ Hogeschool-Universiteit Brussel, Belgium
}

Full addresses

Ceneida Fernández (corresponding autor)

Departamento de Innovación y Formación Didáctica

Facultad de Educación

Campus de San Vicente del Raspeig

03080 Alicante (Spain)

e-mail: ceneida.fernandez@ua.es

Salvador Llinares

Departamento de Innovación y Formación Didáctica

Facultad de Educación

Campus de San Vicente del Raspeig

03080 Alicante (Spain)

e-mail: sllinares@ua.es

Wim Van Dooren

Center for Instructional Psychology and Technology

Katholieke Universiteit Leuven

Vesaliusstraat 2 PB 3770

B-3000 Leuven (Belgium)

email: wim.vandooren@ped.kuleuven.be

Dirk De Bock

Faculty of Economics and Management

Hogeschool-Universiteit Brussel

Stormstraat 2

B-1000 Brussels (Belgium)

email: dirk.debock@hubrussel.be

Lieven Verschaffel

Center for Instructional Psychology and Technology

Katholieke Universiteit Leuven

Vesaliusstraat 2 PB 3770

B-3000 Leuven (Belgium)

email: lieven.verschaffel@ ped.kuleuven.be 


\title{
THE DEVELOPMENT OF STUDENTS' USE OF ADDITIVE AND \\ PROPORTIONAL METHODS ALONG PRIMARY AND SECONDARY \\ SCHOOL
}

\begin{abstract}
This study investigates the development of proportional and additive methods along primary and secondary school. In particular, it simultaneously investigates the use of additive methods in proportional word problems and the use of proportional methods in additive word problems. We have also studied the role played by integer and non-integer relationships between the given numbers and the nature of quantities (discrete or continuous) in the development of these phenomena. A test consisting of additive and proportional missing-value word problems was solved by 755 primary and secondary school students (from fourth to tenth grade). The findings indicate that the use of additive methods in proportional situations increased during primary school and decreased during secondary school whereas the use of proportional methods in additive situations increased along primary and secondary school. Moreover, the presence or absence of integer ratios strongly affects this behaviour but the nature of quantities only has a small influence on the use of proportional methods.
\end{abstract}

Key Words: additive reasoning, multiplicative reasoning, over-use of proportionality, over-use of additive methods, word problems, grade 4 to 10 .

An important achievement in the development of students' numerical thinking is the transition from additive to multiplicative thinking (Harel \& Confrey, 1994; Kieran, 1994; Verschaffel, Greer, \& De Corte, 2007). To a large extent, the acquisition of multiplicative reasoning builds on students' additive reasoning capacities - since multiplication often can be conceived as a repeated addition - but there are also specific aspects that are not reducible to additive models, such as the "Cartesian multiplication" (e.g. "how many different ways of dressing are possible with 5 shirts and 3 trousers?") or the meaning of ratio and rate (e.g. "Robert has driven $45 \mathrm{~km}$ in 38 minutes, how many $\mathrm{km}$ has he driven in 27 minutes if he always kept the same speed?") (Nesher, 1988, 1992; Vergnaud, 1988). As shown in previous research, a characteristic of this development is the students' difficulty to discriminate multiplicative from additive situations (De Bock, Van Dooren, Janssens, \& Verschaffel, 2007; Fernández, Llinares, 
\& Valls, 2008; Modestou \& Gagatsis, 2007; Van Dooren, De Bock, Hessels, Janssens, \& Verschaffel, 2005; Van Dooren, De Bock, Janssens, \& Verschaffel, 2008).

The present study investigates the simultaneous development throughout primary and secondary school of students' use of additive and multiplicative methods in solving additive and multiplicative word problems. In particular, we analyse how primary and secondary school students approach word problems that refer to proportional situations (as a particular case of multiplicative situations) and additive situations. We can clarify the difference between these two kinds of situations by the following examples:

- "Peter and Tom are loading boxes in a truck. They started together but Tom loads faster. When Peter has loaded 40 boxes, Tom has loaded 160 boxes. If Peter has loaded 80 boxes, how many boxes has Tom loaded?"

- "Ann and Rachel are skating around a track. They skate equally fast but Rachel started earlier. When Ann has skated 3 laps, Rachel has skated 12 laps. If Ann has skated 6 laps, how many laps has Rachel skated?"

Both word problems are missing-value problems (where three quantities are given and a fourth is unknown) and in both cases the situation refers to a covariation: The more boxes Peter loaded, the more boxes Tom loaded; the more laps Ann skated, the more laps Rachel skated. However, in the first situation the given numbers are related multiplicatively ( 40 boxes $\times 4=160$ boxes), as the relationship between the boxes loaded by Peter and Tom can be described by a function of the form $f(x)=a x, a \neq 0$. In the second situation the given numbers are linked additively ( 3 laps $+9=12$ laps). The relationship between the laps skated by Ann and Rachel can be modelled by a function of the form $f(x)=x+b, b \neq 0$. 
The first situation can be solved by considering the multiplicative relation between two of the given numbers (ratio between two numbers) and by applying this ratio to the third (proportion as equality of ratios): "Tom has loaded $160: 40=4$ times more boxes than Peter. If Peter has loaded 80 boxes, Tom has loaded $80 \times 4=320$ boxes" (using the external ratio) or "At the second moment, Peter has loaded $80: 40=2$ as many boxes as before. If at the first moment Tom has loaded 160 boxes, then he will have loaded $160 \times 2=320$ boxes at the second moment" (using the internal ratio). The second situation can be solved by considering the additive relation between two of the given numbers (difference between two numbers) and by applying the difference to the third: "Rachel skated 12-3 = 9 laps more than Ann. So if Ann has skated 6 laps, Rachel has skated $6+9=15$ laps". For this reason, in this research, we call the first word problem a "proportional problem" and the second word problem an "additive problem".

Previous research has shown that some students are inclined to use additive methods to solve proportional problems (Hart, 1981; Karplus, Pulos, \& Stage, 1983; Misailidou \& Williams, 2003; Tourniaire \& Pulos, 1985) and, inversely, that some students tend to use proportional methods to solve additive problems (Fernández et al., 2008; Modestou \& Gagatsis, 2007; Van Dooren, De Bock, Evers, \& Verschaffel, 2009). In the following section, we provide some theoretical and empirical background concerning each of these erroneous approaches to additive and proportional word problems.

\section{Theoretical and empirical background}

To study the development of students' approaches to additive and proportional problems along primary and secondary school, three topics from the proportional reasoning literature need further consideration. 
Firstly, there is ample evidence that students of different ages tend to erroneously apply proportional methods to solve different types of non-proportional problems. This points at the difficulty students experience to recognise non-proportional situations and distinguish them from proportional situations (De Bock et al., 2007; Fernández et al., 2008; Van Dooren et al., 2005; Van Dooren et al., 2008). A specific case, which is a focus of the current study, is students' tendency to treat additive situations as if they were proportional. For instance, to the additive problem about two skating girls mentioned above, a large number of students responded erroneously by identifying and using a multiplicative relationship between quantities "As Rachel has skated $12: 3=4$ times more laps than Ann, so when Ann has skated 6 laps, Rachel will have skated $6 \times$ 4 = 24 laps" (Cramer, Post, \& Currier, 1993; Van Dooren et al., 2005). Besides the use of proportional methods in additive situations, another and already older line of research has addressed the incorrect use of additive methods to proportional problems (Hart, 1981; Karplus, Pulos, \& Stage, 1983; Misailidou \& Williams, 2003; Tourniaire \& Pulos, 1985). For example, in the above-mentioned proportional problem about the two boys loading boxes, students could apply an additive method such as "As the difference between the number of boxes loaded by Tom and Peter is $160-40=120$ boxes, Tom will have loaded $80+120=200$ boxes".

In a recent study, Van Dooren, De Bock, and Verschaffel (2010) studied the simultaneous development of these two phenomena (the use of additive methods in proportional situations and the use of proportional methods in additive situations) in primary school students. They observed a decrease from third to sixth grade of the use of additive methods in proportional situations and, at the same time, an increase of the use of proportional methods in additive situations. Their findings indicate a development from applying additive methods (almost) anywhere in the early years of 
primary school to (almost) always applying proportional methods anywhere in the later years of primary school. However, we wonder about what is the development of these phenomena in the transition from primary to secondary school.

Secondly, a well-known issue in the proportional reasoning literature is the fact that the type of ratio affects the use of incorrect additive methods in proportional situations. In particular, non-integer ratios cause more additive errors than integer ratios (Fernández, Llinares, Van Dooren, De Bock, \& Verschaffel, 2009; Harel \& Behr, 1989; Kaput \& West, 1994; Tourniaire \& Pulos, 1985; Van Dooren et al., 2009). However, recently, the role of the type of ratio on the use of incorrect proportional methods in additive situations has been observed too. Van Dooren et al. (2009) and Fernández et al. (2009) studied the effect of non-integer and integer ratios between the given numbers on students' solutions of proportional and additive problems. The proportional problem about the boys loading boxes mentioned before has integer ratios: The external ratio is $160: 40$ and the internal ratio is $80: 40$. Mathematically the same proportional problem but with non-integer ratios could be: "Peter and Tom are loading boxes in a truck. They started together but Tom loads faster. When Peter has loaded 40 boxes, Tom has loaded 100 boxes. If Peter has loaded 60 boxes, how many boxes has Tom loaded?" where the internal ratio is $60: 40$ and the external ratio is $100: 40$. Non-integer versions are more difficult because students cannot identify "the double", "the triple"... In Van Dooren et al.'s study participants were Flemish primary school students (fourth to sixth graders) while Fernández et al. investigated Spanish secondary school students (seventh to tenth graders). In both studies, the non-integer ratios caused more additive errors in proportional problems, and the integer ratios caused more proportional errors in additive problems. From these findings, we wonder about the influence of the type of ratio on the phenomena mentioned above. 
Thirdly, another task characteristic that might affect students' performance on proportional problems is the nature of the quantities involved in the problem. Tourniaire and Pulos (1985) suggested that students can more easily visualize discrete quantities than continuous ones and therefore will perform better on proportional problems involving discrete quantities. However, several later studies have found the opposite. For instance, Jeong, Levine, and Huttenlocher (2007) found that six-, eight-, and tenyear old students more often erroneously used additive counting strategies on proportional problems with discrete quantities and more correct multiplicative strategies on proportional problems with continuous quantities. Also, Boyer, Levine, and Huttenlocher (2008) found that six- to nine-year old children had much more difficulties to solve proportional reasoning problems when the proportions were represented with discrete units than with continuous amounts. However, we have not found research findings about the effect of the nature of quantities on secondary school students' performance on proportional problems and about how this might influence the development of students' methods used to solve these problems. Moreover, the effect of the nature of quantities has not yet been related to the over-use of proportionality, while on the basis of the results of the above-mentioned studies by Tourniaire and Pulos (1985), Jeong et al. (2007) and Boyer et al. (2008), such an effect can be expected.

\section{Research questions}

The study presented here investigates the development of the use of additive methods in proportional situations in parallel with the use of proportional methods in additive situations along primary and secondary school. Also, it investigates the influence of the presence of integer or non-integer ratios between the given numbers and of the nature of quantities on students' approach. We formulate the following research questions: 
- How does students' use of proportional methods in additive problems and of additive methods in proportional problems develop along primary and secondary school?

- How does the number structure (integer vs. non-integer ratios between the given numbers) and the nature of quantities (discrete vs. continuous quantities) affect both developments?

\section{Method}

\section{Participants}

Participants were 755 primary and secondary school students: 65 fourth graders, 68 fifth graders, 64 sixth graders from two different Spanish primary schools and 124 seventh graders, 151 eighth graders, 154 ninth graders, and 129 tenth graders from two different Spanish secondary schools. The participating schools belonged to different cities and students were from mixed socio-economic backgrounds.

Data were collected at the beginning of the academic year 2008-2009. Curriculum contents related to proportional reasoning in the involved age groups are as follows: In the first four years of primary school, there isn't any content related to proportionality, although in grades 3 and 4, the fraction concept appears, as well as equivalent fractions and fraction comparisons. In grades 5 and 6 , the computation of percentages is introduced, as well as the recognition of proportional and non-proportional situations and the use of the "rule of three" algorithm (cross product in a proportion) for proportional situations. According to the curriculum of secondary school, in the seventh grade students should be able to identify proportional quantities from the analysis of data that are organized in tables, to construct a proportion, and to use the rule of three 
algorithm to calculate missing values in proportional problems. The eighth grade introduces inverse proportionality $(f(x)=a / x)$ using corresponding tables and in the ninth and tenth grades the focus lies on the study of linear functions $(f(x)=a x+b)$.

\section{Instrument and procedure}

Students solved a test consisting of eight experimental word problems (four proportional problems $[\mathrm{P}]$ and four additive ones $[\mathrm{A}]$ ), and four buffer problems, all of them formulated in a missing-value format. Buffer problems were included to vary the given tasks and to avoid effects of learning and stereotyped replies.

The design of the experimental items is illustrated in Table 1. To develop these items, we started with eight contexts referring to a discrete variable (e.g. loading boxes in a truck) and eight contexts referring to a continuous variable (e.g. skating a certain distance). Then proportional and additive problems were created by manipulating one sentence (for example, "They started together but Tom loads faster" in the proportional version and "They load equally fast but Tom started earlier" in the additive one). We also manipulated the number structure: integer ratios (when the external and internal ratios are integer: for example $160: 40$ and $80: 40$ in P-D-I in Table 1) and non-integer ratios (when the external and internal ratios are non-integer: for instance $100: 40$ and 60 : 40 in P-D-N in Table 1) between the given numbers. We controlled the number size (we always used one, two or three digits), calculation complexity (the outcome is always an integer), the context (the context is always about actions being performed by two protagonists) and the position of the unknown quantity (when you read the problem the unknown quantity is always in the last position). 
Table 1: Examples of additive $(A)$ and proportional $(P)$ problems with manipulation of the nature of quantities (discrete variable [D] and continuous variables [C]) and the number structure (integer ratios between given numbers [I] and non-integer ratios [N])

\begin{tabular}{|c|c|c|}
\hline Examples & I & $\mathrm{N}$ \\
\hline P-D & $\begin{array}{l}\text { Peter and Tom are loading boxes } \\
\text { in a truck. They started together } \\
\text { but Tom loads faster. When Peter } \\
\text { has loaded } 40 \text { boxes, Tom has } \\
\text { loaded } 160 \text { boxes. If Peter has } \\
\text { loaded } 80 \text { boxes, how many boxes } \\
\text { has Tom loaded? }\end{array}$ & $\begin{array}{l}\text { Peter and Tom are loading boxes } \\
\text { in a truck. They started together } \\
\text { but Tom loads faster. When Peter } \\
\text { has loaded } 40 \text { boxes, Tom has } \\
\text { loaded } 100 \text { boxes. If Peter has } \\
\text { loaded } 60 \text { boxes, how many boxes } \\
\text { has Tom loaded? }\end{array}$ \\
\hline A-D & $\begin{array}{l}\text { Peter and Tom are loading boxes } \\
\text { in a truck. They load equally fast } \\
\text { but Tom started earlier. When } \\
\text { Peter has loaded } 40 \text { boxes, Tom } \\
\text { has loaded } 160 \text { boxes. If Peter has } \\
\text { loaded } 80 \text { boxes, how many boxes } \\
\text { has Tom loaded? }\end{array}$ & $\begin{array}{l}\text { Peter and Tom are loading boxes } \\
\text { in a truck. They load equally fast } \\
\text { but Tom started earlier. When } \\
\text { Peter has loaded } 40 \text { boxes, Tom } \\
\text { has loaded } 100 \text { boxes. If Peter has } \\
\text { loaded } 60 \text { boxes, how many boxes } \\
\text { has Tom loaded? }\end{array}$ \\
\hline P-C & $\begin{array}{l}\text { Ann and Rachel are skating. They } \\
\text { started together but Rachel skates } \\
\text { faster. When Ann has skated } 150 \\
\text { m, Rachel has skated } 300 \text { m. If } \\
\text { Ann has skated } 600 \mathrm{~m} \text {, how many } \\
\text { meters has Rachel skated? }\end{array}$ & $\begin{array}{l}\text { Ann and Rachel are skating. They } \\
\text { started together but Rachel skates } \\
\text { faster. When Ann has skated } 80 \\
\text { m, Rachel has skated } 120 \mathrm{~m} \text {. If } \\
\text { Ann has skated } 200 \mathrm{~m} \text {, how many } \\
\text { meters has Rachel skated? }\end{array}$ \\
\hline A-C & $\begin{array}{l}\text { Ann and Rachel are skating. They } \\
\text { skate equally fast but Rachel } \\
\text { started earlier. When Ann has } \\
\text { skated } 150 \mathrm{~m} \text {, Rachel has skated } \\
300 \mathrm{~m} \text {. If Ann has skated } 600 \mathrm{~m} \text {, } \\
\text { how many meters has Rachel } \\
\text { skated? }\end{array}$ & $\begin{array}{l}\text { Ann and Rachel are skating. They } \\
\text { skate equally fast but Rachel } \\
\text { started earlier. When Ann has } \\
\text { skated } 80 \mathrm{~m} \text {, Rachel has skated } \\
120 \mathrm{~m} \text {. If Ann has skated } 200 \mathrm{~m} \text {, } \\
\text { how many meters has Rachel } \\
\text { skated? }\end{array}$ \\
\hline
\end{tabular}

So, in the test, two proportional problems and two additive problems referred to discrete quantities (for each problem type, one with integer ratios between given numbers [D-I] and the other with non-integer ratios between given numbers [D-N]). The other two proportional problems and the other two additive problems referred to continuous quantities (again two problems had integer ratios $[\mathrm{C}-\mathrm{I}]$ and two problems had noninteger ratios $[\mathrm{C}-\mathrm{N}])$. 
By varying the order of the experimental and buffer problems, we composed a total of eight different tests consisting of eight experimental problems.

Students had 50 minutes (i.e. the duration of a regular math lesson) to complete the test. There were no further test instructions except that students were told that it was allowed to use calculators and were asked to write down their calculations, also if they had done them by means of a calculator.

\section{Analysis}

Students' answers were classified as proportional (Prop, when the answer was achieved by relying on a proportional method), additive (Add, when an additive method was applied to find the answer) and other answer (Oth, when another wrong solution procedure was followed). For proportional problems, proportional methods lead to a correct solution, while for additive problems, additive methods lead to a correct solution. Responses containing purely technical calculation errors (for example $60: 40$ $=1.2$ ) were coded as proportional or additive when the calculations could unequivocally be characterised as such.

Correct answers in proportional and additive problems were scored as 1 and incorrect answers as 0 , in order to carry out a statistical analysis by means of a repeated measures logistic regression analysis, using the generalized estimating of equations (GEE).

\section{Results}

The results section consists of three parts. First, the use of proportional and additive methods along primary and secondary school for each type of problem (proportional or additive) is presented. In the second part, we show how the number structure (the 
presence or absence of integer ratios) influences students' use of proportional and additive methods along primary and secondary school. Finally, we present how the nature of quantities (discrete or continuous quantities) influences students' use of these two methods.

The use of the proportional and additive methods

Figure 1 and Figure 2 show the development of proportional (Prop), additive (Add) and other (Oth) answers for the proportional and additive problems respectively across grades.

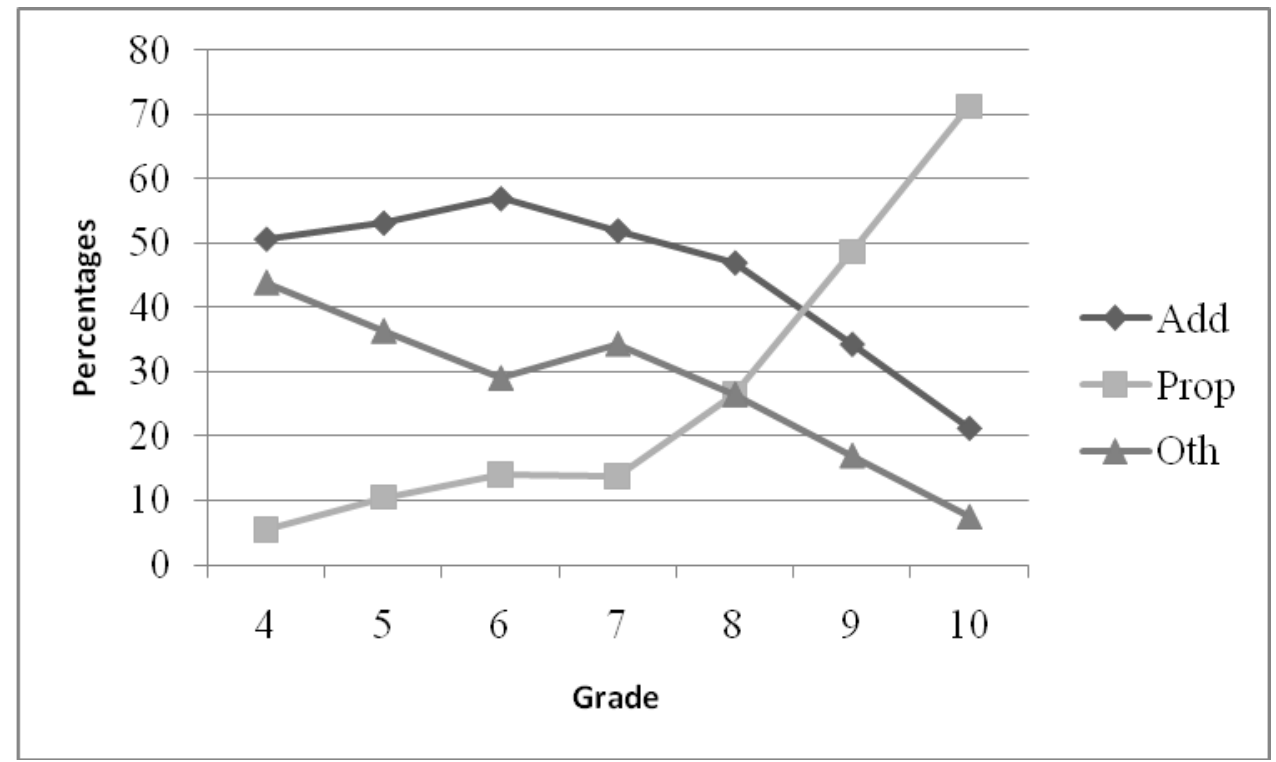

Figure 1. Development of proportional (Prop), additive (Add) and other (Oth) answers on proportional problems from primary to secondary school

For proportional problems the increase of proportional methods is moderate during primary school (fourth to sixth grade), but during secondary school (seventh to tenth grade) it strongly increases coinciding with the introduction of proportionality in the curriculum (Figure 1). Percentages range from $5.5 \%$ in fourth grade to $71.3 \%$ in tenth 
grade. The statistical analysis indicated that differences between grades are significant, $\chi^{2}(6, N=755)=224.509, p<0.001$. Although the differences between fourth and fifth grade $(5.5 \%$ vs. $10.5 \%)$, fifth and sixth grade (10.5\% vs. $14.0 \%)$ and sixth and seventh grade $(14.0 \%$ vs. $13.7 \%)$ are not significant, differences between seventh and eighth grade $(13.7 \%$ vs. $26.5 \%)$, eighth and ninth grade $(26.5 \%$ vs. $48.7 \%)$, and ninth and tenth grade $(48.7 \%$ vs. $71.3 \%)$ are significant.

However, in parallel, there is also a significant increase in the use of incorrect proportional methods to additive problems (Figure 2). Percentages range from $3.0 \%$ in fourth grade to $63.7 \%$ in tenth grade. Differences between fourth and fifth grade $(3.0 \%$ vs. $8.5 \%)$, fifth and sixth grade $(8.5 \%$ vs. $12.7 \%)$ and sixth and seventh grade $(12.7 \%$ vs. $11.0 \%$ ) are not significant. However, differences between seventh and eighth grade ( $11.0 \%$ vs. $20.0 \%)$, eighth and ninth grade $(20.0 \%$ vs. $43.5 \%)$, and ninth and tenth grade $(43.5 \%$ vs. $63.7 \%)$ are significant, $\chi^{2}(6, N=755)=224.509, p<0.001$.

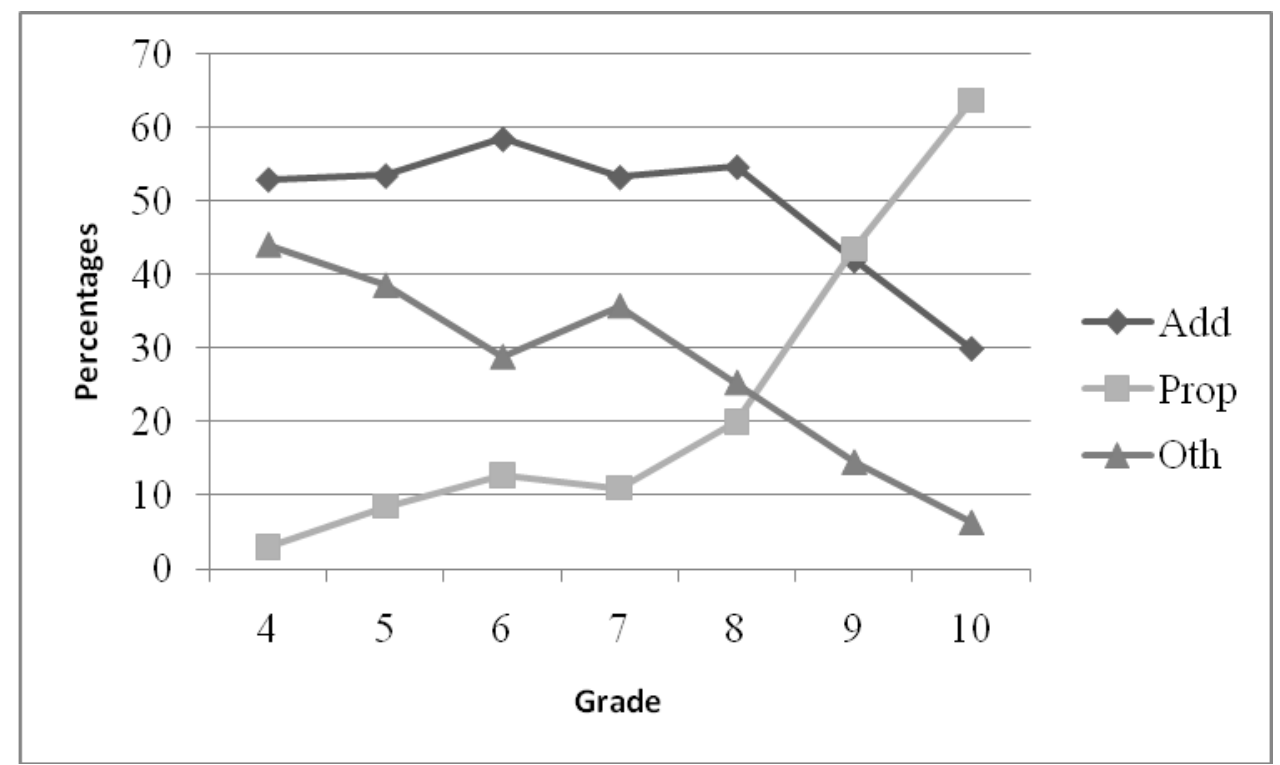

Figure 2. Development of proportional (Prop), additive (Add) and other (Oth) answers on additive problems from primary to secondary school 
Regarding the use of additive methods, there is an increase of additive methods to additive problems during primary school $(53.0 \%$ in fourth grade, $53.0 \%$ in fifth grade, and $58.5 \%$ in sixth grade), but these differences are not significant. But then, there is a decrease during secondary school. While the difference between seventh and eighth grade $(53.3 \%$ vs. $54.7 \%)$ is still not significant, differences between eighth and ninth grade (54.7\% vs. $42.0 \%)$, and ninth and tenth grade (42.0\% vs. $30.0 \%)$ are significant, $\chi^{2}(6, N=755)=15.969, p=0.014$.

However, in parallel, there is an increase of incorrect additive methods to proportional word problems during primary school followed by a decrease during secondary school. The increase along primary school is small and not significant (in fourth grade $50.7 \%$, in fifth grade $53.2 \%$, and in sixth grade $57.0 \%$ ). From seventh to tenth grade, the use of additive methods to proportional word problems decreases. The difference between seventh and eighth grade is not significant $(52.0 \%$ vs. $47.0 \%)$, but the differences between eighth and ninth grade (47.0\% vs. $34.3 \%)$, and ninth and tenth grade $(34.3 \%$ vs. $21.2 \%)$ are stronger and significant, $\chi^{2}(6, N=755)=15.969, p<0.014$.

Finally, the number of other answers decreases along grades, both on proportional and on additive problems. For the proportional problems, differences between fourth and fifth grade (43.8\% vs. $36.3 \%)$, and fifth and sixth grade (36.3\% vs. $29.0 \%)$ are not significant. But differences between seventh and eighth (34.3\% vs. $26.5 \%)$, eighth and ninth $(26.5 \%$ vs. $17.0 \%)$, and ninth and tenth grade $(17.0 \%$ vs. $7.5 \%)$ are significant, $\chi^{2}(6, N=755)=106.614, \mathrm{p}<0.001$. While in the additive problems, differences are not significant between fourth and fifth grade (44.0\% vs. 38.5\%) and fifth and sixth grade (38.5\% vs. $28.8 \%)$, they are significant between seventh and eighth grade $(35.7 \%$ vs. $25.3 \%)$, eighth and ninth grade (25.3\% vs. $14.5 \%)$, and ninth and tenth grade $(14.5 \%$ vs. $6.3 \%)$ 
These data indicate that during primary school (fourth to sixth grade) students mostly use additive methods to additive word problems, but also (incorrectly) to proportional problems. In secondary school (seventh to tenth grade) they switch and mostly use proportional methods to proportional problems, but also (incorrectly) to additive ones.

\section{The influence of the number structure}

Table 2 presents the results of the influence of integer and non-integer ratios on the method used by students in proportional and additive problems along grades.

Table 2. Percentages of the use of proportional and additive methods on proportional and additive problems with integer $(I)$ or non-integer $(N)$ ratios

\begin{tabular}{|c|c|c|c|c|c|c|c|c|c|c|c|c|c|}
\hline \multicolumn{7}{|c|}{ Proportional methods } & \multicolumn{7}{|c|}{ Additive methods } \\
\hline & \multicolumn{3}{|c|}{$\begin{array}{l}\text { Proportional } \\
\text { problems }\end{array}$} & \multicolumn{3}{|c|}{$\begin{array}{l}\text { Additive } \\
\text { problems }\end{array}$} & \multirow[b]{2}{*}{ Grade } & \multicolumn{3}{|c|}{$\begin{array}{c}\text { Proportional } \\
\text { problems }\end{array}$} & \multicolumn{3}{|c|}{$\begin{array}{l}\text { Additive } \\
\text { problems }\end{array}$} \\
\hline Grade & $\mathrm{I}$ & & $\mathrm{N}$ & $\mathrm{I}$ & & $\mathrm{N}$ & & I & & $\mathrm{N}$ & $\mathrm{I}$ & & $\mathrm{N}$ \\
\hline 4 & 10.0 & $>$ & 1.0 & 6.0 & $>$ & 0.0 & 4 & 49.0 & $=$ & 52.5 & 51.5 & $=$ & 54.5 \\
\hline 5 & 20.0 & $>$ & 1.0 & 16.5 & $>$ & 0.5 & 5 & 48.0 & $<$ & 58.5 & 43.5 & $<$ & 62.5 \\
\hline 6 & 22.5 & $>$ & 5.5 & 19.5 & $>$ & 6.0 & 6 & 52.5 & $<$ & 61.5 & 52.0 & $<$ & 65.0 \\
\hline 7 & 22.0 & $>$ & 5.5 & 18.5 & $>$ & 3.5 & 7 & 48.0 & $<$ & 56.0 & 47.5 & $<$ & 59.0 \\
\hline 8 & 33.0 & $>$ & 20.0 & 26.0 & $>$ & 14.0 & 8 & 42.5 & $<$ & 51.5 & 48.5 & $<$ & 61.0 \\
\hline 9 & 55.5 & $>$ & 42.0 & 49.0 & $>$ & 38.0 & 9 & 31.5 & $<$ & 37.0 & 38.5 & $<$ & 45.5 \\
\hline 10 & 74.0 & $>$ & 68.5 & 67.5 & $>$ & 60.0 & 10 & 20.5 & $=$ & 22.0 & 27.0 & $<$ & 33.0 \\
\hline Total & 33.8 & $>$ & 20.5 & 29.0 & $>$ & 17.4 & Total & 41.7 & $<$ & 48.4 & 44.1 & $<$ & 54.4 \\
\hline
\end{tabular}

Along primary and secondary school, students use more proportional methods on integer than on non-integer versions, independently of the proportional or additive character of the problem. This means that integer ratios between given numbers not only led to better performance on proportional problems, but also caused erroneous proportional methods for solving additive problems. For proportional problems $(33.8 \%$ vs. $\left.20.5 \%, \chi^{2}(1, N=755)=75.936, p<0.001\right)$, differences are quite large in grades 4 to 7 , but gradually diminish through grades 8 to 10 (while still remaining significant). For 
additive problems $\left(29.0 \%\right.$ vs. $\left.17.4 \%, \chi^{2}(1, N=755)=57.046, p<0.001\right)$, a very similar development in the use of incorrect proportional methods in relation to the presence of integer or non-integer ratios can be observed.

With respect to the use of additive methods, again, independently of the proportional or additive character of the problems, students use more additive methods for versions with non-integer ratios than for versions with integer ratios. This means that non-integer ratios between given numbers not only led to better performance on additive problems, but also led students to erroneously use additive methods to solve proportional problems. In proportional problems $\left(48.4 \%\right.$ vs. $\left.41.7 \%, \chi^{2}(1, N=755)=69.866, p<0.001\right)$, differences are large and significant in grades 5 to 8 and gradually decrease in grades 9 and 10 (but they are still significant). For additive problems (54.4\% vs. $44.1 \%$ ), $\left.\chi^{2}(1, N=755)=75.936, p<0.001\right)$ there is a similar development in the use of correct additive methods.

\section{The influence of the nature of quantities}

Table 3 presents the results of the influence of the nature of quantities (discrete or continuous) on the method used by students in proportional and additive problems along grades.

Students use slightly more proportional methods on problems with discrete quantities than with continuous ones for both types of problems. For proportional problems, this difference is significant in the whole sample, $\chi^{2}(1, N=755)=5.669, p=0.017$, although it is very small: $27.1 \%$ proportional solutions on the discrete problems vs. only $26.3 \%$ on the continuous problems. The difference, however, is not significant in the different grades. The difference for additive problems is also significant in the whole group, $\chi^{2}(1$, 
$N=755)=8.673, p=0.003$, but very small $(23.9 \%$ vs. $22.6 \%)$ and not significant in the different grades.

Table 3. Percentages of additive and proportional answers to proportional or additive problems with discrete $(D)$ or continuous $(C)$ quantities

\begin{tabular}{|c|c|c|c|c|c|c|c|c|c|c|c|c|c|}
\hline \multicolumn{7}{|c|}{ Proportional methods } & \multicolumn{7}{|c|}{ Additive methods } \\
\hline & \multicolumn{3}{|c|}{$\begin{array}{l}\text { Proportional } \\
\text { problem }\end{array}$} & \multicolumn{3}{|c|}{$\begin{array}{l}\text { Additive } \\
\text { problem }\end{array}$} & \multirow[b]{2}{*}{ Grade } & \multicolumn{3}{|c|}{$\begin{array}{c}\text { Proportional } \\
\text { problems }\end{array}$} & \multicolumn{3}{|c|}{$\begin{array}{l}\text { Additive } \\
\text { problems }\end{array}$} \\
\hline Grade & $\mathrm{D}$ & & $\mathrm{C}$ & $\mathrm{D}$ & & $\mathrm{C}$ & & $\mathrm{D}$ & & $\mathrm{C}$ & D & & $\mathrm{C}$ \\
\hline 4 & 7.0 & $=$ & 4.0 & 3.0 & $=$ & 3.0 & 4 & 50.0 & $=$ & 51.5 & 56.0 & $=$ & 50.0 \\
\hline 5 & 12.5 & $=$ & 8.5 & 9.5 & $=$ & 7.5 & 5 & 51.5 & $=$ & 55.0 & 52.5 & $=$ & 53.5 \\
\hline 6 & 16.5 & $=$ & 11.5 & 14.0 & $=$ & 11.5 & 6 & 53.0 & $=$ & 61.0 & 57.5 & $=$ & 59.5 \\
\hline 7 & 15.0 & $=$ & 12.5 & 12.5 & $=$ & 9.5 & 7 & 52.5 & $=$ & 51.5 & 51.0 & $=$ & 55.5 \\
\hline 8 & 26.0 & $=$ & 27.0 & 20.5 & $=$ & 19.5 & 8 & 47.5 & $=$ & 46.5 & 54.5 & $=$ & 55.0 \\
\hline 9 & 49.0 & $=$ & 48.5 & 44.5 & $=$ & 42.5 & 9 & 35.0 & $=$ & 33.5 & 42.0 & $=$ & 42.0 \\
\hline 10 & 70.5 & $=$ & 72.0 & 63.0 & $=$ & 64.5 & 10 & 22.0 & $=$ & 20.5 & 30.5 & $=$ & 29.5 \\
\hline Total & 27.1 & $>$ & 26.3 & 23.9 & $>$ & 22.6 & Total & 44.5 & $=$ & 45.6 & 49.1 & $=$ & 49.3 \\
\hline
\end{tabular}

Table 3 also indicates that students use only slightly more additive methods on additive problems with continuous quantities $(49.3 \%)$ than on additive problems with discrete quantities (49.1\%). The statistical analysis showed that this difference is not significant.

With respect to the use of additive methods on proportional problems, students use slightly more incorrect additive methods on problems with continuous quantities (45.6\%) than on problems with discrete quantities (44.5\%). But this difference is again not significant.

\section{Conclusions and discussion}

This study focused on the development of the use of additive methods and proportional methods in proportional and additive situations along primary and secondary school. Our results indicate that primary school students mostly use additive methods for additive problems as well as (incorrectly) for proportional word problems. In secondary 
school they start to mostly use proportional methods for proportional problems, but also (incorrectly) for additive problems. So, the use of correct additive methods for additive problems decreases along secondary school, while the use of correct proportional methods for proportional problems increases. Therefore students' performance on proportional problems gets considerably better, but this is not because they become better proportional reasoners (in the sense of being able to distinguish proportional from non-proportional situations). The apparent progress that students make between fourth and tenth grade for proportional problems goes along with a comparable increase of erroneous proportional methods for additive problems. So, it seems that students tend to apply an algorithm learned, rather than trying to reason about the situations at hand (Kaput \& West, 1994).

These findings not only replicate Van Dooren et al.'s (2010) results with Flemish primary school students to Spanish students, but also extend them to secondary school students. Van Dooren et al. suggested that there was a "development from applying additive methods "anywhere" in the early years of primary school to applying proportional methods "anywhere" in the later years" (p. 374) of primary school. Our findings indicated that this claim only holds for Spanish students when we consider the range of age from primary to secondary school. So the observed trends in the development from additive to multiplicative reasoning occur at different ages in each country. Differences in mathematics education traditions in both countries are likely to explain this phenomenon. Generally speaking, the teaching of proportionality comes later in the Spanish curriculum, less attention is paid to discriminating between proportional and non-proportional situations, and the "technical" rule of three algorithm (cross product) takes a more prominent role as the way to solve standard proportional problems. It might be interesting to investigate the simultaneous development of 
additive and proportional reasoning in countries with still other curricular traditions in the teaching of proportionality.

Our results also reveal the influence of two task characteristics in the use of additive and proportional methods, namely of the presence or absence of integer ratios and the nature of the quantities to which a word problem refers. With respect to the first task characteristic, independently of the proportional or additive character of the word problem, students used more proportional methods to problems with integer ratios and used more additive methods to problems with non-integer ratios. As a consequence, students more often use erroneous proportional methods in additive problems with integer ratios, but also more often use correct proportional methods in proportional problems with integer ratios. At the same time, non-integer ratios lead students to use erroneous additive methods in proportional problems, but also to use this method (now correctly) in additive problems. This influence of type of ratio on primary and secondary student's methods used in additive and proportional problems confirms Van Dooren et al.'s (2009) findings and extends them to secondary school students.

With respect to the second task characteristic, our findings indicate that the nature of quantities only affects the trend to use proportional methods, and even that to a very limited extent. Students used slightly more proportional methods to problems with discrete quantities than to problems with continuous ones, independently of the underlying mathematical structure. The low impact of this task variable might be explained by the formulation of the problems in this study in a missing-value format instead of a comparison format, for which stronger effects have been reported in previous research (Boyer et al., 2008; Jeong et al., 2007). Another possible reason refers to the fact that the problems in this study always had an integer outcome. Maybe stronger effects could have been observed too if problems with non-integer outcomes 
were included in the study because such problems can provide a difference in the meaning between continuous and discrete situations. For example, "Peter and Tom are loading boxes in a truck. They started together but Tom loads faster. When Peter has loaded 3 boxes, Tom has loaded 5 boxes. If Peter has loaded 7 boxes, how many boxes has Tom loaded?" In those problems, proportional solutions to discrete problems have little or no sense (e.g., one cannot load $(5 / 3) \times 7=11.6$ boxes in a truck), whereas they are perfectly reasonable for the versions with continuous quantities, as in "Ann and Rachel are skating. They started together but Rachel skates faster. When Ann has skated 10 m, Rachel has skated 15 m. If Ann has skated $21 \mathrm{~m}$, how many meters has Rachel skated?" (In that case, one can skate $(15 / 10) \times 21=31.5$ meters $)$.

Finally, our findings have some implications for educational practice. The fact that there is an increase in the correct use of proportional methods for proportional problems, but also in the incorrect use of these methods for additive problems along primary and secondary school, and the fact that there is a decrease in the use of additive methods along secondary school (even in additive situations where they are correct), implies the necessity to focus students' attention to the analysis of the underlying relationships between quantities in problem situations (Lamon, 1999). Therefore teachers should pay more explicit attention to differences in mathematical structure in order to prevent that students base their solution strategies on superficial associations.

In that sense, Kaput and West (1994) suggest that curricula should also present additive and multiplicative situations jointly to give students opportunities to exercise in discriminating between them, and to focus their attention on the nature of covariation relations. Probably, the use of more realistic situations can also help students to identify and recognize the multiplicative relationships in proportional situations and discriminate them from additive ones. The concrete representations that support and extend students' 
natural build-up reasoning patterns rooted in counting, skip counting, and grouping should be emphasised (Kaput \& West, 1994). Tables and graphs representing proportional and other relationships between quantities in real contexts may further help students to understand these relationships (Lamon, 1995).

\section{References}

Boyer, T. W., Levine, S. C., \& Huttenlocher, J. (2008). Development of proportional reasoning: Where young children go wrong. Developmental Psychology, 44(5), 1478-90.

Cramer, K., Post, T., \& Currier, S. (1993). Learning and teaching ratio and proportion: Research implications. In D. Owens (Ed.), Research ideas for the classroom: Middle grades mathematics (pp. 159-178). New York: Macmillan Publishing Company.

De Bock, D., Van Dooren, W., Janssens, D., \& Verschaffel, L. (2007). The illusion of linearity: From analysis to improvement. New York: Springer.

Fernández, C., Llinares, S., \& Valls, J. (2008). Implicative analysis of strategies in solving proportional and non-proportional problems. In O. Figueras \& A. Sepúlveda (Eds.). Proceedings of the 32nd Conference of the International Group for the Psychology of Mathematics Education (Vol. 3, pp. 1-8). Morelia, México: PME.

Fernández, C., Llinares, S., Van Dooren, W., De Bock, D., \& Verschaffel, L. (2009). Effect of the number structure and the quality nature on secondary school students' proportional reasoning. In M. Tzekaki, M. Kaldrimidou, \& C. Sakonidis (Eds.), Proceedings of the 33rd Conference of the International Group for the Psychology of Mathematics Education (Vol. 3, pp. 25-32). Thessaloniki, Greece: PME. 
Harel, G., \& Behr, M. (1989). Structure and hierarchy of missing-value proportion problems and their representations. Journal of Mathematical Behavior, 8(1), 77-119.

Harel, G., \& Confrey, J. (Eds.) (1994). The development of multiplicative reasoning in the learning of Mathematics. New York: SUNY Press

Hart, K. (1981). Children's understanding of mathematics: 11-16. London: Murray.

Jeong, Y., Levine, S. C., \& Huttenlocher, J. (2007). The development of proportional reasoning: Effect of continuous versus discrete quantities. Journal of Cognition and Development, 8(2), 237-256.

Kaput, J., \& West, M. M. (1994). Missing-value proportional reasoning problems: Factos affecting informal reasoning patterns. In G. Harel \& J. Confrey (Eds.), The development of multiplicative reasoning in the learning of mathematics (pp. 237292). New York: SUNY Press.

Karplus, R., Pulos, S., \& Stage, E. K. (1983). Early adolescents' proportional reasoning on 'rate'. Educational Studies in Mathematics, 14(3), 219-233.

Kieran, T. (1994). Multiple views of multiplicative structure. In G. Harel \& J. Confrey (Eds.), The development of multiplicative reasoning in the learning of mathematics (pp. 387-397). New York: SUNY Press.

Lamon, S. (1999). Teaching fractions and ratios for understanding. Mahwah, NJ: Lawrence Erlbaum Associates.

Lamon, S. (1995). Ratio and proportion: Elementary didactical phenomenology. In J. T. Sowder \& B. P. Schappelle (Eds.), Providing a foundation for teaching mathematics in the middle grades (pp. 167-198). Albany, NY: State University of New York Press. 
Misailidou, C., \& Williams, J. (2003). Diagnostic assessment of children's proportional reasoning. Journal of Mathematical Behavior, 22, 335-368.

Modestou, M., \& Gagatsis, A. (2007). Students' improper proportional reasoning: A result of the epistemological obstacle of "linearity". Educational Psychology, 27(1), $75-92$.

Nesher, P. (1988). Multiplicative school word problems: Theroretical approaches and empirical findings. In J. Hiebert \& M. Behr, (Eds), Number concepts and operations in the middle grades (pp.19-40). Reston, VA: NCTM.

Nesher, P. (1992). Solving multiplication word problems. In G. Leinhardt, R. utman, \& R.A. Hattrup (Eds.), Analysis of aritmetic for mathematics teaching (pp.89-220). Hillsdale, NJ: Lawerence Erlbaum Associates.

Tourniaire, F., \& Pulos, S. (1985). Proportional reasoning: A review of the literature. Educational Studies in Mathematics, 16, 181-204.

Van Dooren, W., De Bock, D., Evers, M., \& Verschaffel, L. (2009). Students' overuse of proportionality on missing-value problems: How numbers may change solutions. Journal for Research in Mathematics Education, 40(2), 187-211.

Van Dooren, W., De Bock, D., \& Verschaffel, L. (2010). From addition to multiplication... and back. The development of students' additive and multiplicative reasoning skills. Cognition and Instruction, 28(3), 360-381.

Van Dooren, W., De Bock, D., Hessels, A., Janssens, D., \& Verschaffel, L. (2005). Not everything is proportional: Effects of age and problem type on propensities of overgeneralization. Cognition and Instruction, 23(1), 57-86. 
Van Dooren, W., De Bock, D., Janssens, D., \& Verschaffel, L. (2008). The linear imperative: An inventory and conceptual analysis of students' overuse of linearity. Journal for Research in Mathematics Education, 39(3), 311-342.

Vergnaud, G. (1988). Multiplicative structures. In J. Hiebert \& M. Behr (Eds.), Number concepts and operations in the middle grades (pp. 141-161). Reston, VA: NCTM.

Verschaffel, L., Greer, B., \& De Corte, E. (2007). Whole number concepts and operations. In F. K. Lester (Ed.), Second handbook of research on mathematics teaching and learning (pp. 557-628). Greenwich, CT: Information Age Publishing. 